\title{
Rare presentation of a gaint coronary artery aneurysm as a mediastinal mass
}

\author{
Madhu Andrew Philip, MCh, Korah Kuruvila Thomas, MCh, Ravi Shankar, MCh, \\ Biswa Ranjan Panda, MCh \\ Department of Cardiothoracic Surgery, Christian Medical College, Vellore, Tamil Nadu, India
}

This 26-year-old female presented with chest pain for 2 months. Based on her chest X-ray (Fig. 1.1) and Computed Tomogram Thorax, (Fig 1.2) she was referred to us as a case of a mediastinal mass compressing the right atrium. A 64 slice Computed Tomogram showed a large aneurysm arising from the proximal part of the right coronary artery (Fig. 1.3).

Surgical findings were, a large aneurysmal mass measuring about $6 \times 4$ inches with the main right coronary artery stretching over it before entering the aneurysmal mass. The aneurysm was opened (Fig. 1.4). Clots within were evacuated. No distal lumen of the vessel was seen. The main right coronary artery was opening into the roof of the aneurysm which was ligated. Then an aortocoronary bypass to the posterior descending branch of the right coronary artery was done.

The patient had an uneventful post-operative period. There was no fresh Electrocardiogram changes post operatively.

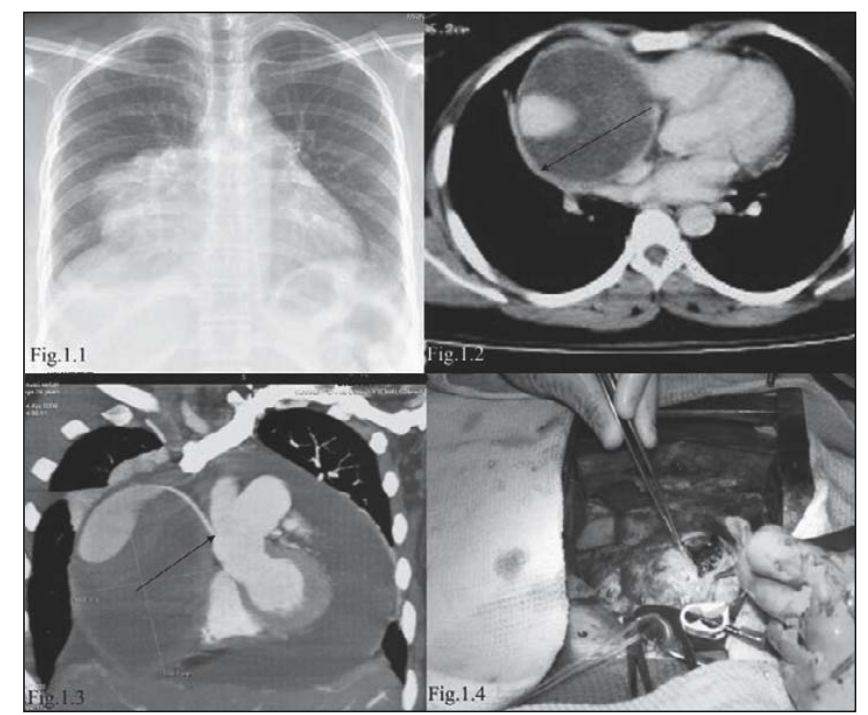

Fig. 1.1 - Chest X-ray showing a probable mediastinal mass 1.2 Computed Tomogram Thorax showing right paracardiac mass, probably a right coronary artery aneurysm, the right coronary artery is seen arching over the mass (arrow). 1.3 - 64 slice Computed Tomogram showing the mass to represent a partly thrombosed aneurysm arising from the right coronary artery, the artery is seen arising from the root of the aorta (arrow) and entering this aneurysm 1.4 - Intraoparative image showing the aneurysm filled with clots.

Address for correspondence:

Dr. Korah Kuruvila Thomas

Department of Cardiothoracic Surgery

Christian Medical College

Vellore, Tamil Nadu, India, 632004

Phone : 0416-2282106, 0416-2282160, 0416-2282884

Facsimile numbers : 0416-2282035

E-mail : k_kuruvila@yahoo.co.in

(C) IJTCVS 097091342520609/41 IM

Received - 02/04/09; Review Completed - 09/04/09; Accepted - 11/04/09. 